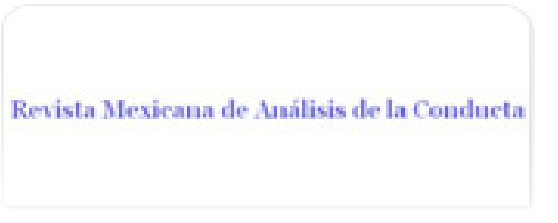

Revista Mexicana de Análisis de la Conducta ISSN: 0185-4534

editora@rmac-mx.org

Sociedad Mexicana de Análisis de la Conducta México

VALLE CHAUVET, CARLOS FELIPE DEL; HERNÁNDEZ-POZO, MARÍA DEL ROCÍO EFECTO DE UNA INTERVENCIÓN PSICOLÓGICA BREVE SOBRE LA EJECUCIÓN FUTBOLÍSTICA PROFESIONAL

Revista Mexicana de Análisis de la Conducta, vol. 36, núm. 1, junio, 2010, pp. 97-114 Sociedad Mexicana de Análisis de la Conducta

Guadalajara, México

Disponible en: http://www.redalyc.org/articulo.oa?id=59313134007

- Cómo citar el artículo

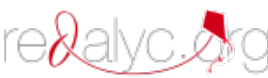

- Número completo

- Más información del artículo

- Página de la revista en redalyc.org

Sistema de Información Científica

Red de Revistas Científicas de América Latina, el Caribe, España y Portugal Proyecto académico sin fines de lucro, desarrollado bajo la iniciativa de acceso abierto 


\title{
EFECTO DE UNA INTERVENCIÓN PSICOLÓGICA BREVE SOBRE LA EJECUCIÓN FUTBOLÍSTICA PROFESIONAL
}

\author{
DIFFERENTIAL EFFECT OF A BRIEF PSYCHOLOGICAL TRAINING ON \\ PERFORMANCE OF PROFESSIONAL FOOTBALL SOCCER PLAYERS \\ CARLOS FELIPE DEL VALLE CHAUVET ${ }^{1 *} Y$ \\ MARÍA DEL ROCÍO HERNÁNDEZ-POZO ${ }^{2}$ \\ 1PROGRAMA DE DOCTORADO, FACULTAD DE PSICOLOGÍA, \\ UNIVERSIDAD NACIONAL AUTONOMA DE MEXICO \\ 2PROYECTO APRENDIZAJE HUMANO, UNIVERSIDAD NACIONAL \\ AUTONOMA DE MEXICO, FES IZTACALA
}

\begin{abstract}
Resumen
El objetivo de la presente investigación fue determinar si una intervención psicológica breve favorecería categorías específicas de rendimiento futbolístico en un equipo de futbolistas profesionales de primera división en México. Participaron 28 jugadores, 11 titulares y 17 suplentes, en un programa de 6 sesiones de entrenamiento que incluyó: relajación, imaginería, control del diálogo interno y solución de problemas. Se registró una mejora en acciones defensivas, cambio de juego y faltas para el grupo titular $(F=4.177, p=0.012$; $F=3.3635, p=0.029 ; F=19.6333, p<.0001$, respectivamente), y para acciones ofensivas y cambio de juego en el grupo suplente $(F=5.6219, p=0.004 ; F=3.21$, $p=0.035$, respectivamente). Se concluye que una intervención breve de ese tipo, beneficia de forma diferencial a titulares y suplentes en tres clases particulares de acciones futbolísticas y que estos efectos positivos no se explican por la sola participación en los juegos de la liga.

Palabras Clave: categorías conductuales, registro observacional, futbolistas profesionales, rendimiento deportivo, intervención psicológica breve.
\end{abstract}

\footnotetext{
*Este artículo es un reporte de la investigación doctoral del primer autor, bajo la dirección de la segunda autora. Agradecemos las observaciones de los revisores anónimos que ayudaron a mejorar sustancialmente el escrito. Dirigir la correspondencia a María de Rocío Hernanndez Pozo, PIAH, cubiculo 5, piso 2, Edificio UliCSE, FES Iztacala, Universidad Nacional Autónoma de México, Ave. vallesch@yahoo.com y herpoz@unam.mx

Recibido: Febrero 2, 2010 


\section{Abstract}

The purpose of this study was to assess whether a brief psychological intervention would affect specific categories of performance in a professional team of first division soccer leagues in Mexico. 28 athletes, 11 titular and 17 substitute, participated in a 6 session program which included relaxation techniques, imagery, control of internal dialogue and problem solving. A significative improvement for the titular group was recorded in defensive actions, change of game, and penalties ( $F=4.177, p=0.012 ; F=3.3635$, $p=0.029 ; F=19.6333, p<.0001$, respectively), while the substitute group increased its offensive and change of game actions $(F=5.6219, p=0.004$; $F=3.21, p=0.035$, respectively). This study concludes that a brief intervention of this sort benefits differentially in three classes of actions both groups of soccer players, and that this positive effects are not explained by the mere participation in the league games of the professional season.

Key Words: behavioral categories, observational record, soccer professional players, sport performance, brief psychological intervention.

Desde comienzos de la década de los setentas se ha registrado un incremento en las investigaciones sistemáticas en Psicología del Deporte (Cruz, 1997), esta disciplina se ha caracterizado por una orientación más programática que conceptual, ya que se han dirigido la mayor parte de sus esfuerzos al ámbito competitivo y a la metodología de entrenamiento. Martens (1987) menciona: "nuestra finalidad es ayudar a los deportistas a mejorar sus habilidades psicológicas para poder hacer frente a las presiones asociadas a la competencia de alto nivel" (pag. 69).

Los especialistas del deporte trabajan en dilucidar las variables que permiten que los atletas tengan un mejor control de si mismos, autorregulen sus emociones, pensamientos y sensaciones, de modo que les permitan elevar su calidad deportiva y manejar adecuadamente los eventos disposicionales y estímulos distractores presentes en las situaciones de competencia. El entrenamiento psicológico constituye un aspecto relevante dentro del entrenamiento atlético integral.

Las líneas de investigación más populares en Europa y Estados Unidos, gravitan alrededor de cuatro áreas de investigación que incluyen: a) aumentar del rendimiento deportivo de atletas de elite; b) disminuir el tiempo en la recuperación de lesiones; c) desarrollar estrategias para la adherencia al entrenamiento deportivo en personas sedentarias, de la tercera edad, o con problemas de salud, y d) caracterizar los estilos de comportamiento exitoso en deportistas de diferentes disciplinas. 
Diversos investigadores han documentado el papel que juegan elementos psicológicos como la motivación, la cohesión de equipo y las tendencias de comportamiento sobre el desempeño atlético, y de una manera central la forma en que la atención y el control del estrés afectan el rendimiento deportivo (Buceta, 1999; Sánchez \& Lejeune, 1999; Passos \& Gouveia, 1999; Ruiz, 1999; Mora, Zarco \& Blanca, 2001; Larumbe, 2001; Nieto \& Olmedilla, 2001; Fierro, 2002; Buceta, 2002; Noce \& Samulski, 2002; Ortín, 2003; Baker, Coté \& Abernethy, 2003; Rodríguez, Wigfield \& Eccles, 2003; Voight, 2005).

En el contexto del fútbol de elite el rendimiento competitivo es multidimensional, debido a la contribución de factores que lo afectan. Frecuentemente se sostiene que un rendimiento óptimo depende de un grado alto de desarrollo y especialización en cuatro dimensiones: física, técnica, táctica y la psicológica (Garganta, 1997).

A diferencia de lo que sucede en otras modalidades consideradas de dominancia energético funcional, o de carácter técnico combinado, en el fútbol los componentes táctico y psicológico parecen condicionar de forma importante el rendimiento del jugador (Konzag, 1983; Teodorescu, 1984; Castelo, 1994; Bayer, 1994; Mombaerts, 2000; Gréhaigne, 2001).

Destrezas en el manejo de la atención y el control de estrés, centrales ambas para las dimensiones táctica y psicológica del rendimiento deportivo pueden aprenderse, desarrollarse y perfeccionarse, al igual que ocurre con las destrezas físicas y técnicas. Algunos estudios reportados en la literatura especializada en este campo han demostrado la eficacia de técnicas psicológicas con atletas, para mejorar las capacidades atencionales y el control del estrés dentro de la práctica deportiva (Martín \& Hrycaiko, 1983; Garfield \& Bennett, 1984).

Varios estudiosos señalan la necesidad de estudiar los procesos psicológicos de los futbolistas dentro de un contexto social deportivo (Martens 1979; Gill, 1986; Caracuel, 1993); estudios con esa perspectiva han arrojado resultados positivos en disciplinas como atletismo (Spink, 1988; Spink 1990), hockey (Halliwell, 1990; Nideffer, 1991), tenis (McPherson \& French, 1991) y en otros deportes de equipo (Davis, 1988).

Los atletas que participan en deportes competitivos necesitan emplear habilidades psicológicas y estrategias de afrontamiento que les permita satisfacer sus expectativas y mejorar su rendimiento (Márquez, 2006), así como autorregular sus emociones, en especial aquellas que pueden poner en peligro su rendimiento, como el estado de ánimo, la falta de motivación, el exceso de ansiedad y preocupaciones y la falta de concentración (Velásquez \& Guillén, 2007). 
Mora, García, Toro y Zarco (1995; 2000) describen la aplicación de un programa para mejorar las estrategias cognitivas con jugadores profesionales de futbol, basada en las teorías sobre el estilo atencional (Nideffer 1976; 1991). En ese estudio se aplicó un programa enfocado a mejorar la atención para cuatro tipos de acciones en la cancha, definidas como: controles, anticipaciones, intercepciones y faltas cometidas. Lamentablemente en ese estudio los autores no encontraron diferencias significativas entre el grupo control y el experimental, quizá debido a la ausencia de ejercicios prácticos.

El fútbol a nivel mundial es uno de los deportes que más se practica y que cuenta con más espectadores, algo similar sucede en México. La Federación Mexicana de Futbol (FMF) señaló que durante el año, más de 950,000 personas acudieron a los estadios para presenciar en vivo los últimos 20 partidos en los que participó la selección nacional, adicionalmente los promedios de audiencia televisiva por partido de la selección mexicana ha sido de 11 millones en un universo de 38 millones de televidentes a nivel nacional (Agencia EFE, 2007).

De esto se deriva que los jugadores al ser fácilmente identificables por la afición, tienen una alta probabilidad de experimentar presión social. Esto aunado a las contingencias normales a que están sujetos los atletas profesionales, en donde hay una relación directa entre relaciones sus resultados y los ingresos y el prestigio, contribuye a un entramado de condiciones que pueden generar estrés que ocasione falta de concentración y bajo rendimiento.

Una revisión de la literatura especializada nos lleva a concluir que en Latinoamérica existen pocas investigaciones científicas relacionados con el tema, existiendo un enorme vacío en el área.

Dado que el ámbito futbolístico profesional es de difícil acceso, y no se presta a investigaciones académicas, el objetivo del presente estudio fue medir el efecto de una intervención breve, sin evaluaciones individuales previas, sobre categorías específicas de ejecución futbolística previamente usadas por otros autores (Mora, García, Toro \& Zarco, 2000), a partir de un estudio observacional apoyado en filmaciones de los partidos.

La hipótesis era que el rendimiento deportivo mejoraría en todos los atletas, independientemente de posibles diferencias individuales no medidas, o de su clasificación deportiva, y que el efecto de la intervención se mantendría al menos durante una temporada, es decir, en los siguientes nueve partidos a partir de haber recibido el entrenamiento.

La investigación se centró en evaluar cambios en las frecuencias de acciones futbolísticas defensivas, ofensivas, cambio de juego defensivo-ofensivo y faltas cometidas, producto del entrenamiento psicológico, independientemente de otros factores que pudieran ejercer influencia sobre el desempeño deportivo, partiendo del supuesto que la exposición a esas herramientas psicológicas, ayudaría a minimizar los distractores, dentro y fuera de la cancha que pudieran obstaculizar el rendimiento futbolístico. 


\section{Método}

El carácter central del estudio fue observacional, ya que se evaluó el comportamiento deportivo a partir de categorías de acciones futbolísticas, con un solo grupo integrado por el total de miembros de un equipo, que constó de dos subgrupos, titulares y suplentes, que recibieron un entrenamiento grupal corto de 9 horas. Antes y después de la intervención, se registraron categorías conductuales basadas en la codificación interjueces del desempeño individual, a partir de filmaciones de un total de 36 partidos.

\section{Participantes}

Se trabajó con un grupo de 28 jugadores profesionales de fútbol, de entre 18 y 30 años de edad, todos pertenecientes al mismo equipo de primera división. Los participantes tenían una edad promedio de 22.5 años, así como una edad competitiva promedio de 5 años en el circuito profesional. Todos estaban registrados ante la Federación Mexicana de Fútbol, y pertenecían al equipo de primera división profesional bajo estudio. 11 jugadores formaban parte del grupo titular y 17 eran suplentes, esa asignación a los subgrupos se hizo como es costumbre en los equipos de este deporte, siguiendo criterios técnicos no explícitos del entrenador o jefe técnico deportivo, tendientes a seleccionar a los elementos más prometedores que aumentarían la probabilidad de éxito del equipo. Los jugadores del grupo suplente, son aquellos que ingresan a un partido en sustitución de un jugador titular, para aumentar las probabilidades estratégicas de obtener puntuación en un encuentro, siguiendo el plan técnico del entrenador responsable.

\section{Aparatos y Materiales}

Se utilizó un instrumento de observación constituido por formatos de campo y sistemas de categorías (Ardá \& Anguera, 1999) que se reproduce en el Anexo 1. Para definir e identificar los patrones de juegos se construyó un sistema de cuatro macro-categorías asociadas al papel ofensivo o defensivo del jugador.

Macro-categoría 1: Construcción y desarrollo del proceso ofensivo. En este rubro las categorías fueron: a) Recepción y control del balón, b) Evitación del adversario.

Macro-categoría 2: Construcción y desarrollo del proceso defensivo. Se subdividió en: a) Anticipación al contrario, b) Apoyo a dos contra uno (cuando un compañero apoya a la defensa a otro en la marcación de un contrario), y c) Recuperación del terreno de juego.

Macro-categoría 3: Cambio de juego defensivo-ofensivo, constituida por las intercepciones.

Macro-categoría 4: Faltas cometidas. 
Tanto el registro como las codificaciones se realizaron a partir del análisis de las cintas grabadas de los partidos. Cada uno de los partidos se grabó en video, mediante tres cámaras, para su análisis posterior. Las cintas empleadas fueron las que las televisoras proporciona después de cada juego; por lo general de un mismo partido se les dan al equipo tres cintas que corresponden a cámaras diferentes, una en el centro de la cancha y las otras dos detrás de cada portería.

Se analizaron los primeros 18 partidos de dos torneos, es decir, un total de 36 partidos. Tres jueces entrenados realizaron la observación, dos de éstos eran los analistas del equipo, que eran expertos en los elementos a evaluar, siendo ellos los que entrenaron al tercer juez, el primer autor de esta investigación. El entrenamiento en el registro observacional concluyó hasta que las observaciones alcanzaron una confiabilidad interjueces e intertomas de cámaras, igual o superior al $90 \%$, basada en el registro a partir de los formatos de campo y sistemas de categorías descritos.

En el instrumento de observación se anotó el número de ocurrencias de cada una de las siete categorías conductuales, de acuerdo con los criterios ya definidos, de todos los partidos que se jugaron durante dos temporadas, y ese registro se hizo de manera individual para cada jugador. Cada 15 minutos se usó una hoja de observación diferente.

Durante la intervención, adicionalmente al registro conductual por categorías se emplearon dos tipos de tablas, una de planes de re-enfoque (Buceta, 1994), en las cuales el deportista hacía un plan de su propio comportamiento para el partido siguiente, y una tabla de objetivos, donde los jugadores escribían sus objetivos individuales asociados al objetivo grupal, organizados en una matriz de 3 por 2 celdillas, con plazos largo, medio y corto por dos tipo de objetivo, de producto o resultado y de proceso.

\section{Procedimiento}

La investigación se desarrolló durante los primeros 18 partidos de dos torneos nacionales de apertura, por lo que el estudio abarcó 36 partidos distribuidos en dos años consecutivos.

El primer torneo que abarcó los partidos 1 al 18, compuesto por las temporadas 1 y 2 de 9 partidos cada una, se caracterizó por la ausencia de programa psicológico dirigido a los participantes, ausencia de observadores y de psicólogo. Los datos individuales de cada futbolista en esa etapa se registraron de acuerdo a las categorías observacionales antes descritas.

El segundo torneo, que constó de los partidos 19 al 36, se dividió en dos temporadas, la 3 y 4 . La temporada 3 se consideró como línea base (partidos 19 a 27), durante la cual los jugadores se percataron que eran observados y percibieron también la presencia del psicólogo del equipo. Después del partido 27 y antes del partido 28 se programó la intervención psicológica breve, que se administró de manera grupal durante seis sesiones de aproximadamente 90 minutos cada una. 
Todo el equipo participó en el entrenamiento, incluyendo jugadores de banca y los que sólo actuaron en los partidos de preparación o partidos interescuadras; esta estrategia no obedeció a un plan metodológico del estudio, sino mas bien a una exigencia del entrenador.

En la fase de intervención se entrenaron habilidades consistentes en plantear objetivos, usar auto-instrucciones, controlar la respiración, relajación e imaginería, con la finalidad de mejorar el control atencional, proporcionar herramientas para el manejo de estrés y neutralizar la influencia que pudiera ejercer el público sobre el rendimiento individual del jugador.

Durante la temporada 4 del torneo, es decir en los partidos 28 al 36, se tomaron medidas para evaluar el efecto del programa. Se midió el impacto que tuvo la intervención sobre el rendimiento de los jugadores, a través del cambio en acciones técnicas concretas durante los partidos; al final de esta fase se aplicó un cuestionario de satisfacción con el programa.

\section{Resultados}

Los resultados se organizaron en función de las categorías de registro, para las diferentes fases del estudio, antes, durante y después de la intervención, para cada grupo, el de titulares con 11 integrantes y el de suplentes con 17.

\begin{tabular}{|c|c|c|c|c|c|c|c|c|c|c|c|c|c|c|c|c|c|c|c|c|}
\hline \multirow{2}{*}{$\begin{array}{c}\text { Titulares } \\
\text { \#/temporada }\end{array}$} & \multicolumn{4}{|c|}{$\begin{array}{l}\text { Partidos } \\
\text { jugados }\end{array}$} & \multicolumn{4}{|c|}{$\begin{array}{l}\text { Media anticipaciones } \\
\text { por partido }\end{array}$} & \multicolumn{4}{|c|}{$\begin{array}{l}\text { Media controles } \\
\text { por partido }\end{array}$} & \multicolumn{4}{|c|}{$\begin{array}{l}\text { Media intercepciones } \\
\text { por partido }\end{array}$} & \multicolumn{4}{|c|}{$\begin{array}{l}\text { Media falta } \\
\text { por partido }\end{array}$} \\
\hline & 1 & 2 & 3 & 4 & 1 & 2 & 3 & 4 & 1 & 2 & 3 & 4 & 1 & 2 & 3 & 4 & 1 & 2 & 3 & 4 \\
\hline $1 \mathrm{~T}$ & 9 & 9 & 9 & 3 & 24.6 & 25.2 & 26.6 & 24.6 & 21.6 & 22.5 & 21.5 & 19.3 & 9.8 & 7.5 & 6.4 & 4.3 & 2.7 & 1.4 & 1.7 & 0.3 \\
\hline $2 \mathrm{~T}$ & 9 & 7 & 9 & 9 & 25.4 & 24.1 & 25.5 & 31.3 & 24.1 & 21.5 & 26.4 & 23.2 & 7.1 & 5.7 & 6.4 & 8.3 & 3.3 & 1.7 & 2.0 & 1.3 \\
\hline $3 \mathrm{~T}$ & 9 & 9 & 9 & 2 & 24.3 & 27.7 & 27.6 & 22.5 & 28.6 & 21.8 & 23.5 & 26.0 & 7.2 & 6.4 & 4.7 & 7.0 & 3.4 & 1.5 & 1.1 & 2.0 \\
\hline $4 \mathrm{~T}$ & 9 & 9 & 8 & 9 & 24.8 & 25.7 & 26.5 & 29.0 & 25.8 & 20.4 & 23.3 & 24.3 & 5.5 & 8.2 & 5.0 & 8.5 & 3.2 & 1.1 & 1.8 & 1.5 \\
\hline $5 \mathrm{~T}$ & 7 & 8 & 6 & 9 & 27.8 & 26.1 & 26.1 & 30.8 & 25.7 & 21.8 & 28.5 & 27.7 & 5.8 & 6.3 & 6.6 & 6.7 & 2.7 & 0.7 & 1.0 & 1.3 \\
\hline $6 \mathrm{~T}$ & 9 & 8 & 9 & 9 & 25.8 & 28.3 & 23.7 & 27.6 & 24.3 & 22.7 & 24.5 & 25.4 & 6.7 & 7.8 & 6.3 & 8.5 & 4.2 & 1.6 & 1.7 & 1.0 \\
\hline $7 \mathrm{~T}$ & 9 & 6 & 8 & 4 & 23.6 & 24.3 & 21.7 & 27.2 & 24.2 & 19.3 & 23.3 & 26.7 & 6.8 & 8.0 & 5.1 & 8.0 & 2.7 & 1.6 & 2.3 & 1.0 \\
\hline $8 \mathrm{~T}$ & 7 & 8 & 8 & 4 & 22.0 & 23.3 & 24.8 & 27.2 & 25.2 & 24.5 & 25.0 & 17.7 & 8.8 & 5.7 & 5.0 & 8.2 & 3.0 & 1.0 & 1.6 & 0.7 \\
\hline 9T & 9 & 9 & 0 & 0 & 23.0 & 22.1 & & & 26.0 & 23.0 & & & 4.8 & 6.6 & & & 3.2 & 1.8 & & \\
\hline $10 \mathrm{~T}$ & 9 & 8 & 4 & 7 & 24.3 & 21.8 & 27.2 & 25.5 & 23.4 & 20.7 & 23.7 & 24.4 & 7.3 & 5.8 & 6.0 & 8.4 & 2.7 & 1.8 & 0.7 & 1.4 \\
\hline $11 \mathrm{~T}$ & 7 & 8 & 0 & 1 & 21.1 & 21.7 & & 28.0 & 23.0 & 21.6 & & 21.0 & & 7.8 & & 6.0 & 5.7 & 3.7 & & 0.0 \\
\hline
\end{tabular}

La Tabla 1 muestra el número de partidos jugado por cada futbolista titular durante las 4 temporadas, así como las medias por temporada de acciones defensivas/anticipaciones; acciones ofensivas/de recepción y control; cambio de juego de defensivo a ofensivo/intercepciones, y faltas por partido jugado. 
Tabla 2.

Partidos jugados durante las cuatro temporada (9 partidos), media de anticipaciones, controles, intercepciones y faltas cometidas por partido por temporada, por cada jugador suplente.

\begin{tabular}{|c|c|c|c|c|c|c|c|c|c|c|c|c|c|c|c|c|c|c|c|c|}
\hline \multirow{2}{*}{$\begin{array}{l}\text { Sustitutos } \\
\# / \text { temporada }\end{array}$} & \multicolumn{4}{|c|}{$\begin{array}{l}\text { Partidos } \\
\text { jugados }\end{array}$} & \multicolumn{4}{|c|}{$\begin{array}{l}\text { Media anticipaciones } \\
\text { por partido }\end{array}$} & \multicolumn{4}{|c|}{$\begin{array}{l}\text { Media controles } \\
\text { por partido }\end{array}$} & \multicolumn{4}{|c|}{$\begin{array}{l}\text { Media intercepciones } \\
\text { por partido }\end{array}$} & \multicolumn{4}{|c|}{$\begin{array}{l}\text { Media falta } \\
\text { por partido }\end{array}$} \\
\hline & 1 & 2 & 3 & 4 & 1 & 2 & 3 & 4 & 1 & 2 & 3 & 4 & 1 & 2 & 3 & 4 & 1 & 2 & 3 & 4 \\
\hline $12 \mathrm{~S}$ & 6 & 8 & 6 & 0 & 19.0 & 24.1 & 24.0 & & 22.6 & 19.2 & 22.0 & & 5.1 & 7.7 & 4.3 & & 2.1 & 2.1 & 1.3 & \\
\hline $13 \mathrm{~S}$ & 3 & 3 & 6 & 1 & 15.6 & 20.0 & 24.6 & 28.0 & 23.6 & 22.3 & 22.0 & 28.0 & 6.3 & 3.6 & 5.6 & 12.0 & 2.3 & 1.0 & 1.5 & 2.0 \\
\hline $14 \mathrm{~S}$ & 2 & 1 & 0 & 0 & 17.5 & 25.0 & & & 17.5 & 22.0 & & & 5.0 & 9.0 & & & 4.5 & 1.0 & & \\
\hline $15 \mathrm{~S}$ & 4 & 0 & 0 & 0 & 21.5 & & & & 19.7 & & & & 4.2 & & & & 2 & & & \\
\hline $16 \mathrm{~S}$ & 4 & 2 & 6 & 9 & 22.7 & 15.5 & 22.8 & 25.8 & 15.5 & 14.5 & 22.1 & 24.0 & 6.2 & 5 & 4.5 & 7.4 & 2.2 & 0.5 & 0.8 & 1.7 \\
\hline $17 \mathrm{~S}$ & 2 & 3 & 0 & 0 & 3.5 & 16.6 & & & 4.0 & 13.0 & & & 2.0 & 3.0 & & & 1.5 & 0.0 & & \\
\hline $18 \mathrm{~S}$ & 1 & 9 & 1 & 0 & 18.0 & 17.1 & 9.0 & & 11.0 & 11.6 & 28 & & 4.0 & 5.3 & 9.0 & & 0.0 & 1.4 & 1.0 & \\
\hline $19 \mathrm{~S}$ & 0 & 2 & 4 & 8 & & 14.5 & 24.0 & 25.5 & & 12.0 & 20.5 & 25.0 & & 8.0 & 5.7 & 9.6 & & & 2.5 & 0.8 \\
\hline $20 \mathrm{~S}$ & 0 & 0 & 8 & 8 & & & 23.8 & 25.2 & & & 19.8 & 27.3 & & & 5.6 & 8.3 & & & 1.3 & \\
\hline $21 \mathrm{~S}$ & 0 & 0 & 3 & 6 & & & 33.7 & 21.3 & & & 23.5 & 26.0 & & & 6.7 & 8.6 & & & 2.0 & 1.1 \\
\hline $22 \mathrm{~S}$ & 0 & 0 & 2 & 8 & & & 20.0 & 21.7 & & & 17.0 & 26.5 & & & 3.0 & 10.2 & & & 1.0 & 1.3 \\
\hline $23 \mathrm{~S}$ & 0 & 0 & 1 & 1 & & & & & & & & & & & & & & & & \\
\hline $24 \mathrm{~S}$ & 0 & 0 & 4 & 9 & & & 24.5 & 26.1 & & & 19.5 & 23.0 & & & 4.5 & 8.8 & & & 2.0 & 1.1 \\
\hline $25 \mathrm{~S}$ & 0 & 0 & 4 & 4 & & & 21.2 & 20.0 & & & 21.0 & 24.0 & & & 6.0 & 6.0 & & & 1.7 & .5 \\
\hline $26 \mathrm{~S}$ & 0 & 0 & 0 & 1 & & & & 24.0 & & & & 19.0 & & & & 1.0 & & & & \\
\hline $27 \mathrm{~S}$ & 0 & 0 & 0 & 1 & & & & 24.0 & & & & 28 & & & & 8.0 & & & & \\
\hline $28 \mathrm{~S}$ & 0 & 0 & 0 & 3 & & & & 18.6 & & & & 21.3 & & & & 10.0 & & & & 2.0 \\
\hline
\end{tabular}

Siguiendo la misma lógica, la Tabla 2, presenta los datos de los jugadores suplentes, que participaron de manera muy heterogénea en las cuatro temporadas, por ejemplo sólo 3 de los 17 suplentes jugaron en las 4 temporadas, y en ese mismo grupo, 4 solo jugaron en una temporada. Los futbolistas titulares jugaron en promedio 28 partidos de 36 que incluyó el estudio, en comparación con una media de 9 partidos para los jugadores suplentes.

Tabla 3.

Comparación estadística entre grupos titular y suplente, con base en las medianas de anticipaciones, controles, intercepciones y faltas por temporada por grupo.

\begin{tabular}{|c|c|c|c|c|}
\hline & \multicolumn{4}{|c|}{ Temporadas } \\
\hline & 1 & 2 & 3 & 4 \\
\hline Anticipaciones & $\begin{array}{c}\text { mediana } \\
(\mathrm{n})\end{array}$ & $\begin{array}{c}\text { mediana } \\
(\mathrm{n})\end{array}$ & $\begin{array}{c}\text { mediana } \\
(\mathrm{n})\end{array}$ & $\begin{array}{c}\text { mediana } \\
(\mathrm{n})\end{array}$ \\
\hline Titular & $24.3(11)$ & $24.3(11)$ & $26.1(9)$ & $27.4(10)$ \\
\hline Suplente & $18.0(7)$ & $17.1(7)$ & $23.9(10)$ & 24.0 (11) \\
\hline \multirow{3}{*}{$\begin{array}{r}\text { Diferencia } \\
\text { entre grupos }\end{array}$} & $t=3.704$ & $t=3.726$ & $\mathrm{t}=1.322$ & $\mathrm{t}=3.051$ \\
\hline & $\mathrm{gl} .=16$ & $\mathrm{gl} .=16$ & $\mathrm{gl}=17$ & $\mathrm{gl} .=16$ \\
\hline & $\mathrm{p}=0.0019$ & $\mathrm{p}=0.0018$ & $\mathrm{p}=0.2037$ & $\mathrm{p}=0.0066$ \\
\hline Controles & $\begin{array}{l}\text { mediana } \\
\text { (n) }\end{array}$ & $\underset{(\mathrm{n})}{\operatorname{mediana}}$ & $\begin{array}{l}\text { mediana } \\
(\mathrm{n})\end{array}$ & $\underset{(\mathrm{n})}{\operatorname{mediana}}$ \\
\hline Titular & $24.3(11)$ & $21.8(11)$ & $23.7(9)$ & $24.3(10)$ \\
\hline Suplente & $17.5(7)$ & $14.5(7)$ & $21.5(10)$ & $25.0(11)$ \\
\hline Diferencia & $t=3.896$ & $t=3.672$ & $\mathrm{t}=2.507$ & $\mathrm{t}=-0.852$ \\
\hline \multirow[t]{2}{*}{ entre grupos } & $\mathrm{gl}=16$ & $\mathrm{gl} .=16$ & $\mathrm{gl} .=17$ & $\mathrm{gl}=19$ \\
\hline & $\mathrm{p}=0.0013$ & $\mathrm{p}=0.0021$ & $\mathrm{p}=0.0226$ & $\mathrm{p}=0.4046$ \\
\hline Intercepciones & $\begin{array}{l}\text { mediana } \\
\text { (n) }\end{array}$ & $\begin{array}{c}\text { mediana } \\
(\mathrm{n})\end{array}$ & $\begin{array}{l}\text { mediana } \\
\text { (n) }\end{array}$ & $\begin{array}{c}\text { mediana } \\
(\mathrm{n})\end{array}$ \\
\hline Titular & $7(10)$ & $6.6(11)$ & $6.0(9)$ & $8.1(10)$ \\
\hline Suplente & $5(7)$ & $5.3(7)$ & $5.6(10)$ & $8.6(11)$ \\
\hline Diferencia & $t=3.168$ & $\mathrm{t}=1.254$ & $\mathrm{t}=0.407$ & $\mathrm{t}=-0.781$ \\
\hline \multirow[t]{2}{*}{ entre grupos } & $\mathrm{gl}=15$ & $\mathrm{gl} .=16$ & $\mathrm{gl}=17$ & $\mathrm{gl}=19$ \\
\hline & $\mathrm{p}=0.0064$ & $\mathrm{p}=0.2278$ & $\mathrm{p}=0.6888$ & $\mathrm{p}=0.4443$ \\
\hline Faltas & $\begin{array}{c}\text { mediana } \\
(\mathrm{n})\end{array}$ & $\begin{array}{c}\text { mediana } \\
\text { (n) }\end{array}$ & $\begin{array}{c}\text { mediana } \\
(\mathrm{n})\end{array}$ & $\begin{array}{c}\text { mediana } \\
(\mathrm{n})\end{array}$ \\
\hline \multirow{5}{*}{$\begin{array}{r}\text { Titular } \\
\text { Suplente } \\
\text { Diferencia } \\
\text { entre grupos }\end{array}$} & $3.2(11)$ & $1.6(11)$ & $1.7(9)$ & $1.1(10)$ \\
\hline & $2.1(7)$ & $1.0(7)$ & $1.4(10)$ & $1.5(11)$ \\
\hline & $t=2.452$ & $\mathrm{t}=1.381$ & $\mathrm{t}=0.242$ & $\mathrm{t}=-1.840$ \\
\hline & $\mathrm{gl}=16$ & $\mathrm{gl}=16$ & $\mathrm{gl}=17$ & $\mathrm{gl} .=19$ \\
\hline & $\mathrm{p}=0.0261$ & $\mathrm{p}=0.1864$ & $\mathrm{p}=0.8118$ & $\mathrm{p}=0.0814$ \\
\hline
\end{tabular}


NTERVENCIÓN PSICOLÓGICA BREVE SOBRE LA EJECUCIÓN FUTBOLÍSTICA

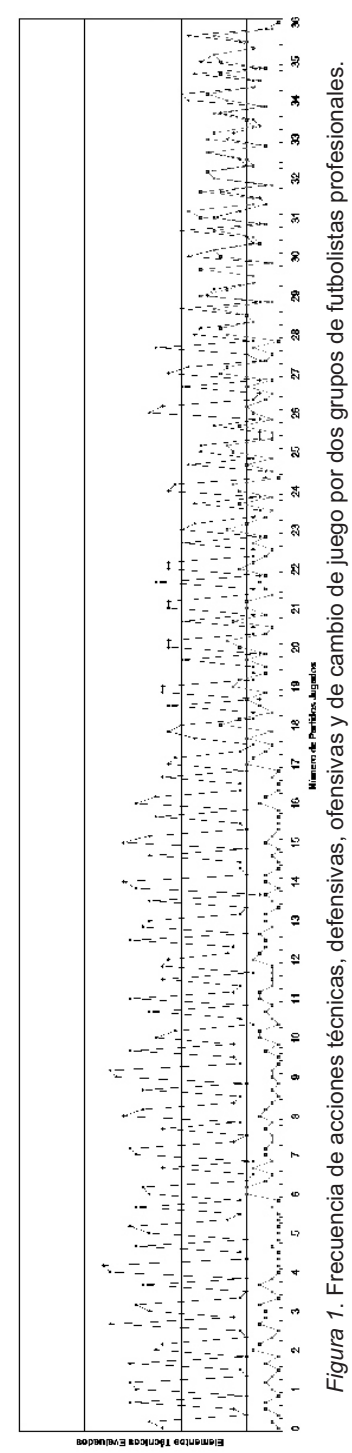


En la Tabla 3 se presentan las medianas para cuatro categorías conductuales seleccionadas para ilustrar con más detalle los cambios diferenciales para titulares y suplentes, registrados después de la intervención. Entre paréntesis se muestra la cifra de jugadores de cada grupo cuyos datos se presentan desglosados por temporadas. La prueba t arrojó diferencias significativas entre grupos titular y suplente, a favor de los primeros en todas las categorías de la primera temporada, y en anticipaciones y controles para la segunda temporada, también favoreciendo a los titulares. En la tercera temporada, hubo cambios que tuvieron como consecuencia la desaparición de diferencias significativas entre grupos, en tres de las acciones, posiblemente debido a la presencia del psicólogo y al ser observadas y analizadas las acciones deportivas de los futbolistas de manera individual. En la cuarta temporada tampoco se registraron diferencias entre titulares y suplentes en tres de cuatro acciones deportivas.

En la Figura 1 se presenta la frecuencia de 6 conductas: defensivas, ofensivas y de cambio de juego, durante los dos torneos, ó 4 temporadas que comprenden 36 partidos; de ellos se distinguen los primeros 18 partidos por la ausencia de observadores, psicólogo y programas de intervención, de los últimos 18 partidos que si contaron con observadores, psicólogo y por que entre las temporadas 3 y 4 los futbolistas fueron expuestos al programa de intervención. Los datos secuenciales se presentan en función de los grupos titular y suplente. La curva superior desde el inicio del registro corresponde a la ejecución de los titulares, mientras que el polígono de frecuencia que durante el primer torneo es bajo y posteriormente sube a partir del partido 28 , corresponde a la ejecución de los futbolistas suplentes.

Se puede observar en esta figura que los jugadores titulares mostraron un nivel superior al de los suplentes en las fases iniciales del registro, de hecho los polígonos de frecuencia no se tocan para ambos grupos durante el primer torneo (partidos 1 al 18).

En el segundo torneo (partidos 19 al 36), la frecuencia de conductas deseables por parte de los jugadores titulares disminuye de manera no significativa y la de los suplentes aumenta ligeramente, asociada al inicio del programa en el partido 19, en que se contabas con la presencia de observadores. A partir del partido 28, justo después de la aplicación del programa psicológico, los suplentes comienzan a mejorar las conductas técnicas deseables, tendencia que se consolida a partir del partido 31 y que se establece hasta llegar a igualar e incluso superar en algunos juegos, la ejecución de jugadores titulares, en las categorías registradas. El grupo titular, en la temporada 4, que corresponde a los partidos 28 al 36, muestra una disminución moderada no significativa en la frecuencia de conductas deportivas positivas en los encuentros, en comparación con la ejecución en la temporada previa (partidos 19 al 27). 


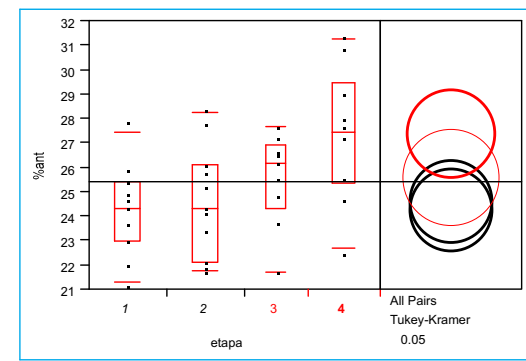

Figura 2. Media de anticipaciones para el grupo de futbolistas titulares durante 4 temporadas

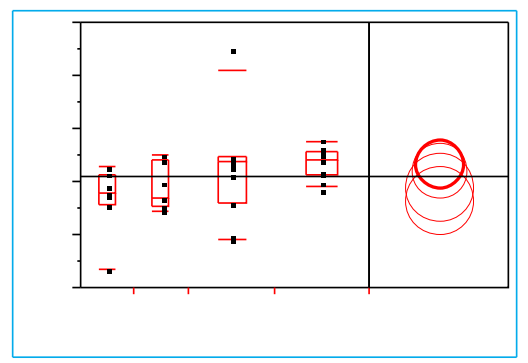

Figura 3. Media de anticipaciones para el grupo de futbolista suplentes durante 4 temporadas

Las figuras 2 a la 9 presentan diagramas de caja que sirven para ilustrar de manera detallada la distribución de las medias de las conductas de anticipación, controles, intercepciones y faltas cometidas respectivamente, para los grupos titular y suplente durante las 4 temporadas analizadas. En estas figuras, en la parte derecha se representa visualmente las diferencias entre grupos mediante círculos, de acuerdo con la metodología del Análisis Exploratorio de Datos. Los futbolistas titulares incrementaron de manera significativa las acciones defensivas de anticipación, en la fase posterior a la intervención $(F=4.177, g l=40, p=0.0121)$. El incremento en el grupo suplente para esta misma categoría conductual, no fue significativo $(F=2.052, g l=34$, $p=0.1309)$, como se muestra en la Figura 3 .

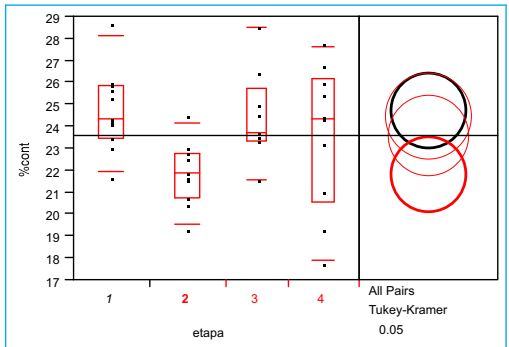

Figura 4. Media de recepciones o controles para el grupo titular de futbolistas en las 4 temporadas

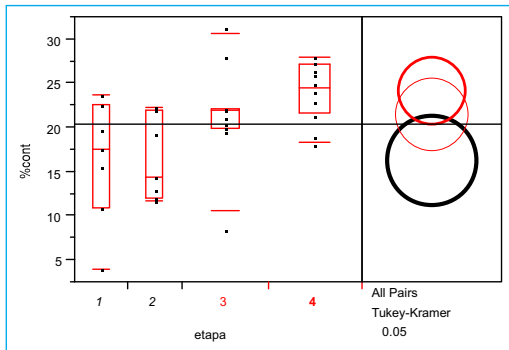

Figura 5. Media de recepciones o controles para los futbolistas suplentes en las 4 temporadas

Con respecto a las acciones ofensivas de controlar el balón, los futbolistas titulares iniciaron la primera temporada con una tendencia ofensiva alta, que bajó de manera sustancial en la segunda temporada y luego volvió a subir. En la cuarta temporada, después de la intervención, los titulares no mostraron un incremento homogéneo de acciones ofensivas, sino más bien, un amplio rango de estas acciones. Las diferencias en conductas medias por temporadas o etapas fueron significativas para los titulares $(F=3.7076, g l=40, p=0.0199)$, 
pero no se produjo una tendencia regular unidireccional.

En la Figura 5 que corresponde al grupo suplente, se registraron cambios significativos a la alza para los futbolistas, después de la intervención $(F=5.6219, g l=34, p=0.0042)$. El efecto positivo de la intervención fue muy claro para este grupo.

Las figuras 6 y 7 muestran las intercepciones para el grupo titular y para el suplente respectivamente.

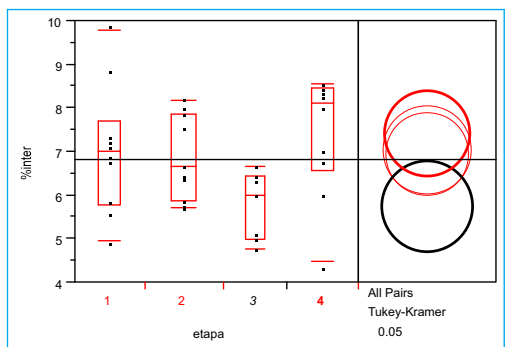

Figura 6. de intercepciones para el grupo titular de futbolistas en las 4 temporadas

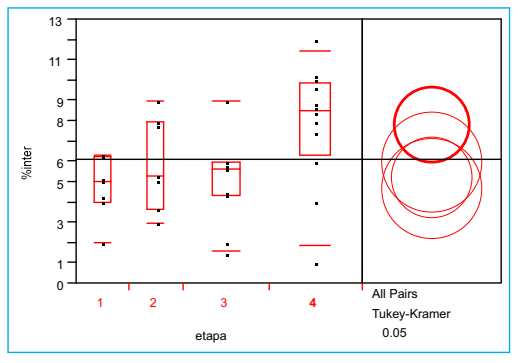

Figura 7. Media de intercepciones para los futbolistas suplentes en las 4 temporadas

En ambos casos después de la intervención psicológica se produjo el cambio esperado, esto es, un aumento en este tipo de acciones. El cambio conductual para los jugadores titulares, ocurrió en la dirección esperada y fue significativo $(F=3.3635, g l=39, p=0.0291)$, y para el grupo suplente también se registró la misma tendencia $(F=3.2124, g l=34, p=0.0354)$.

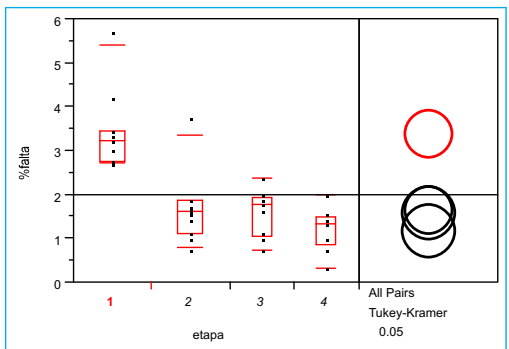

Figura 8. Media de faltas cometidas para el grupo titular de futbolistas en las 4 temporadas

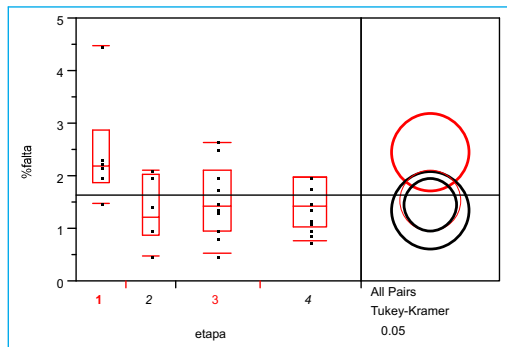

Figura 9. Media de faltas cometidas para los futbolistas suplentes en las 4 temporadas

Finalmente las faltas cometidas para cada grupo se presentan en las figuras 8 y 9 , para titulares y suplentes. En ambos casos bajó la frecuencia de faltas en el transcurso de las temporadas 1 a 4, siendo significativas las diferencias por temporada para cada grupo, el titular $(F=19.6333, g l=40, p<.0001)$ y el suplente $(F=3.4824, g l=34, p=0.0279)$, aunque las diferencias para los suplentes entre las temporadas 3 y 4 fueron marginales. 
Se esperaría que durante las temporadas 2 y 4 las acciones futbolísticas de los integrantes al interior de cada subgrupo titular o suplente, fueran similares, debido a que esas temporadas representan condiciones contingenciales con demandas equivalentes, esto es, en ambos casos, los jugadores están expuestos al efecto acumulativo de 9 primeros partidos del año, en los cuales el equipo se enfrenta a un número de rivales que constituyen desafíos secuenciales similares. De ese modo la hipótesis nula con respecto al efecto de la intervención sería que no habría cambios entre las temporadas 2 y 4 , y si los hubiera, sería muy probable que éstos se debieran a la intervención administrada entre la temporada 3 y la 4.

Las acciones futbolísticas durante la temporada 4, al ser comparadas para cada subgrupo con las registradas durante la segunda temporada, ponen en evidencian el efecto benéfico de la intervención, para el grupo titular, en acciones anticipatorios $(t=2.567, g l=19, p=0.0189)$. La misma comparación para el grupo suplente arrojo dos cambios de mejora, en las acciones de anticipaciones y de controles ( $t=2.86, g l=17, p=.0108$ y $t=4.23, g l=17$, $p=.0006$; respectivamente).

Por lo que respecta a la valoración del programa por parte de los futbolistas, ésta resultó positiva en cuanto a su apreciación del beneficio personal, de cara a la práctica deportiva profesional. Los futbolistas consideraron útil y necesario contar con herramientas de ese tipo.

En conclusión, los ejercicios psicológicos que se pusieron al alcance de los futbolistas profesionales, tuvieron efectos benéficos desde un punto de vista estadístico, para ambos grupos, titular y suplente, para la categoría de cambio de juego defensivo a ofensivo. Se registraron adicionalmente efectos diferenciales estadísticamente significativos para el grupo titular, consistentes en un incremento de las acciones defensivas, así como en la disminución de faltas cometidas. En contraste, el grupo suplente experimentó un incremento en sus acciones técnicas ofensivas, como consecuencia de la intervención.

\section{Discusión}

El propósito de este estudio se concentró en examinar el efecto de una intervención psicológica grupal corta, dirigida a futbolistas profesionales de un equipo de primera división, sin mediar evaluaciones preliminares conductuales de los jugadores, con base en un análisis cuantitativo detallado de siete aspectos técnicos del desempeño futbolístico a partir de filmaciones de partidos durante dos torneos anuales.

Los resultados observados en cuanto a la mejoría de los jugadores titulares y suplentes, para categorías conductuales específicas, ilustra un efecto diferencial previamente señalado en la literaria deportiva, en función de grado de experiencia de los atletas. Tretilova y Rodimiki (1979) documentaron la diferencia entre expertos y novatos al comparar los ritmos cardiacos de 22 
tiradores con menor experiencia, y mostraron que los primeros eran capaces de controlar su tasa cardiaca óptima mientras realizaban el tiro, mientras que los segundos eran incapaces de hacerlo; de igual forma Fenz (1975) realizó una investigación similar con paracaidistas encontrando que con los novatos la tasa cardiaca por minuto se incrementaba desde el primer momento del salto y hasta tocar tierra, a diferencia de los paracaidistas experimentados, que mostraban un incremento de la tasa cardiaca al principio, pero poco después esta comenzaba a disminuir y gradualmente decrecía hasta lograr la tasa que tenían antes del salto. Mahoney y Avener (1977) encontraron un efecto en el mismo sentido cuando trabajaron con gimnastas olímpicos, observando que los no seleccionados presentaban niveles de activación iguales que los seleccionados, y sin embargo al momento de competir los segundos lograban controlar la ansiedad y bajar el nivel de activación.

En el presente estudio los resultados indican que los titulares exhibieron las habilidades psicológicas óptimas que han ido desarrollando a lo largo de su carrera deportiva, no obstante, el programa fue beneficioso para que ese grupo incrementara sus acciones defensivas y redujera las faltas. Por otro lado mediante el programa de entrenamiento psicológico, se mostró evidencia de que se puede acelerar el proceso de aprendizaje y dominio de las habilidades ofensivas y de cambio de juego defensivo a ofensivo en atletas con menos experiencia, que en principio serían equivalentes funcionalmente a las obtenidas por los titulares durante una exposición prolongada a las contingencias deportivas.

Esto sugiere que una de las diferencias entre los jugadores titulares y los suplentes, radica básicamente en que los primeros muestran mayor capacidad para controlar el estrés que los segundos, y no la magnitud del estrés a que están expuestos antes y durante los partidos. El estudio permitió observar que los jugadores suplentes, como consecuencia del programa de intervención, pudieron controlar mejor el estrés antes y durante un partido y fueron capaces de mostrar un mejor nivel de juego ofensivo; ese cambio positivo en el desempeño deportivo del grupo suplente, pudo estar relacionado con la disminución del efecto interferente que ejercen elementos distractores y eventos generadores de estrés.

Estos resultados coinciden con lo reportado en un estudio en que se dio entrenamiento en atención a un grupo de 36 deportistas universitarios, en el cual se encontró que el entrenamiento en atención focalizada o dividida, mejoró el desempeño de atletas de cuatro modalidades de deporte de pelota, que incluyeron el fútbol, el baloncesto, el fútbol de salón y el voleibol (Rodríguez \& Montoya, 2006). 
En el mismo sentido, Brignani (1989) también obtuvo resultados en los que se aprecia una mejora técnico-táctica en la mayor parte de los jugadores, como resultado de sesiones semanales de entrenamiento atencional en una situación real de juego de voleibol.

A pesar de que las sesiones de trabajo fueron breves, aproximadamente de nueve horas de entrenamiento grupal, consideramos que la sencillez de las técnicas permitió que los futbolistas las aprendieran y se consolidara su uso en la práctica diaria.

Los hallazgos de esta investigación alientan el diseño de programas cortos centrados en el entrenamiento de la atención y control del estrés para mejorar el rendimiento deportivo en equipos profesionales de fútbol, ya que aún con una intervención tan breve como la administrada en este estudio, sin controles de laboratorio, ni mediciones individuales del perfil psicológico de los participantes, se registraron efectos significativos evidentes en varias de las categoría conductuales observadas intrasujeto y entre grupos.

Se sabe que otros factores pueden jugar un papel específico en el desempeño futbolístico, como lo han señalado algunos autores (Geisler \& Leith, 2007), entre los cuales no se descarta un componente social de la "atmósfera" de juego, pero los resultados de este estudio destacan el papel central que juega el aprendizaje de técnicas psicológicas para mejorar la atención y controlar el estrés.

\section{Referencias}

Agencia EFE. (2007, Febrero 10). México a punto del millón de aficionados reunidos en estadios en el 2007. Hoy en los Deportes. Recuperado Marzo 29 del 2009, http://mx.sports.yahoo.com/10092007/38/deportes-m-xicopunto-mill-n-aficionados-reunidos-estadios-2007.html

Ardá, T., \& Anguera, M. (1999). Observación de la acción ofensiva en fútbol 7. Utilización del análisis secuencial de la identificación de patrones de juego ofensivos. En Angueram M. (Coord). Observación en deporte y conducta cinético motriz. Aplicaciones. Barcelona: Universitat de Barcelona.

Baker, J., Cóte, J., \& Abernethy, B. (2003). Sport-specific practice and the development of expert decision-making in team ball sports. Journal of Applied Sport Psychology, 15(1), 12-25.

Bayer, C. (1994). La enseñanza de los deportes colectivos.Barcelona: Hispano-Europea.

Brignani, D. (1989). Metodologia per l'allenamento dell'attenzione nella pallavolo. Tesis ISEF dell'Aquila e Scuola dello Sport di Roma.

Buceta, J. M. (1994). La intervención psicológica para la optimización del rendimiento. En J. Gil \& M. A. Delgado (eds.) Psicología y Pedagogía de la Actividad Física y el Deporte. Madrid: Siglo XXI.

Buceta, J. M. (1999). Variables psicológicas relacionadas con el rendimiento físico y deportivo. España: UNED. 
Buceta, J. M. (2002). Intervención psicológica con corredores de maratón: características y valoración del programa aplicado en el maratón de Madrid. Revista de Psicología del Deporte, 11 (1), 83-110.

Caracuel, J. C. (1993). Relaciones entre psicología básica y psicología del deporte: Una interacción fructífera. Revista de Psicología General y Aplicada, 46 (3), 339-346.

Castelo, J. (1994). Fútebol. Modelo técnico-táctico do jogo. Lisboa: edicoes $\mathrm{FMH}$

Cruz, J. (Ed.). (1997). Psicología del Deporte. Madrid: Síntesis.

Davis, J. (1988). Strategies for managing athletes jet lag. Sport Psychologist, $1(2), 154-160$.

Fenz, W. D. (1975). Strategies for coping with stress. In I. G. Sarason \& C. D. Spielberger (Eds.) Stress \& Anxiety, vol. 2, Washington; Hemisphere and Publishing Corporation.

Fierro, G. (2002). Variables relacionadas con el éxito deportivo en ligas NBA y ACB de baloncesto. Revista de Psicología del Deporte, 11 (2), 247-258.

Garfield, C. A., \& Bennett, H. Z. (1984). Rendimiento Máximo. Barcelona: Martínez Roca.

Garganta, J. (1997). Modelacao táctica do jogo de futebol. Estudo da organizao da fase ofensiva em equipas de alto rendimiento. Tesis doctoral no publicada. Porto: Universidade de Porto.

Gill, D. (1986). Psychological dynamics of sport. Champaign, II.: Juman Kinetics.

Gréhaigne, J. F. (2001). La organización del juego en el fútbol. Barcelona: INDE

Geisler, G., \& Leith, L. M. (2007). Estados motivacionais de jogadores de futebol no Canadá, Alemanha e Japão na perspectiva da teoria dos reversos. Revista Brasileira de Psicologia do Esporte, 1(1), 1-19.

Halliwell, W. (1990). Providing sport psychology consulting services in professional Hockey. Sport Psychologist, 4, 369-377.

Konzag, I. (1983). La formazione tecnico-tattica nei giochi sportivi. Revista di Cultura Sportiva, 11 (2), 42-47.

Larumbe, E. (2001). Entrenamiento de variables psicológicas para los tiros libres. Revista de Psicología del Deporte, 10 (1), 89-87.

Mahoney, M. J., \& Avener, M. (1977). Psychology of the athlete: An exploratory study. Cognitive Therapy and Research, 1, 135-141.

Márquez, S. (2006). Estrategias de afrontamiento del estrés en el ámbito deportivo: Fundamentos teóricos e instrumentos de evaluación. International Journal of Clinical and Health Psychology, 6 (2), 359-378.

Martens, R. (1979). About smocks and jocks. Journal of Sport Psychology, 1 , 94-99. 
Martens, R. (1987). Coaches Guide to Sport Psycholgy. Champaign, III.: Human Kinetics.

Martín, G. L., \& Hrycaiko, D. (1983). Effective behavioral coaching. What's all about? Journal of Sport Psychology, 5, 8-20.

McPherson, L., \& French, E. (1991). Changes in cognitive strategies and motor skill in tennis. Journal of Sport and Exercise Psychology, 13 (1), 26-41.

Mombaerts, E. (2000). Fútbol. Del análisis del juego a la formación del jugador. Barcelona: INDE.

Mora, J. A., García, J., Toro, S., \& Zarco, J. A. (1995). Estrategias cognitivas en Deportistas Profesionales. Málaga: Coedición SPICUM y Dirección General de Deportes.

Mora, J. A., García, J., Toro, S., \& Zarco, J. A. (2000). CECD. Cuestionario de Estrategias Cognitivas en Deportistas. Madrid: TEA Ediciones.

Mora, J. A., Zarco, J. A., \& Blanca, M. J. (2001). Atención-Concentración como entrenamiento para la mejora del rendimiento deportivo en jugadores profesionales de fútbol. Revista de Psicología del Deporte, 10 (1), 49-65.

Nideffer, R. M. (1976). The inner athlete: Mind plus Muscle for winning. Nueva York: Thomas Crowell.

Nideffer, R. M. (1991). Entrenamiento para el control de la atención y la concentración. En J. M. Williams (Ed.), Psicología Aplicada al Deporte. Madrid: Biblioteca Nueva.

Nieto, G., \& Olmedilla, A. (2001). Planificación del entrenamiento psicológico en atletas de elite: Un caso en marcha atlética. Revista de Psicología del Deporte, $10(1), 127-142$.

Noce, F., \& Samulski, D. M. (2002). Análisis del estrés psíquico en colocadores brasileños de voleibol de alto nivel. Revista de Psicología del Deporte, 11(1), 137-156.

Ortín, F. J. (2003). La utilización de registros para la mejora del comportamiento táctico en deporte de equipo. Revista de Psicología del Deporte, 12(1), 95106.

Passos, P., \& Gouveia L. (1999). Entrenamiento en imaginería con motociclistas. Revista de Psicología del Deporte, 8(1), 133-146.

Rodríguez, D., Wigfield, A., \& Eccles, J. (2003). Changing competence perceptions, changing values: Implications for youth sport. Journal of Applied Sport Psychology, 15(1), 67-81.

Rodríguez, M. C., \& Montoya, J. C. (2006). Entrenamiento en la atención en deportistas y su efectividad en el rendimiento. Acta Colombiana de Psicología, 9(1), 99-112.

Ruiz, L. (1999). Rendimiento deportivo, optimización y excelencia en el deporte. Revista de Psicología del Deporte, 8(2), 235-248. 
Sánchez, X., \& Lejeune, M. (1999). Práctica mental y deporte: ¿Qué sabemos después de casi un siglo de investigación? Revista de Psicología del Deporte, 8(1), 21-37.

Spink, K.S. (1988). Facilitating endurance performance; The effects of cognitive strategies and analgesic suggestions: The Sport Psychology, 2, 97-104.

Spink, K.S. (1990). Collective efficacy in the sport setting. International Journal of Sport Psychology, 21, 4, 380-395.

Teodorescu, L. (1984). Problemas de teoría e metodogia nos jogos desportivos. Lisboa: Livros Horizonte.

Tretilova, T. A., \& Rodimiki, E. M. (1979). Investigation of emotional state of rifle shooters. Theory and Practice of Physical Culture, 39, 5-28.

Velásquez, M. T., \& Guillén, N. (2007) Regulación emocional en nadadores en proceso de formación deportiva, categoría infantil.Avances Latinoamericanos de Psicología, 25 (2), 112-125.

Voight, M. (2005). Integrating mental-skills training into everyday coaching. Journal of Physical Education, Recreation \& Dance, 7(3), 38-47. 